\section{Kognitive Veränderungen unter Anastrozol bei Brustkrebs nach der Menopause}

\author{
Zu den kognitiven Veränderungen unter adjuvanter Aromatasehemmer- \\ therapie existieren nur wenige prospektive Studien. Aromatasehemmer \\ bewirken postmenopausal einen fast kompletten Entzug von Östradiol, was \\ mit einer Verschlechterung kognitiver Funktionen einhergehen kann.
}

\begin{abstract}
n eine große Kohorten-Verlaufsstudie wurden postmenopausale Frauen mit frühem Brustkrebs im Stadium I-IIIa aufgenommen, die entweder Chemotherapie plus Anastrozol $(\mathrm{n}=114)$ oder nur Anastrozol $(n=173)$ erhielten. Ihnen wurde eine Gruppe altersentsprechender Frauen $(\mathrm{n}=110)$ gleichen Bildungsniveaus ohne Brustkrebs als Kontrolle gegenübergestellt. Alle Frauen wurden neurokognitiven Tests vor der systemischen Therapie sowie 6, 12 und 18 Monate danach unterzogen. In der Kontrollgruppe wurden die Tests zu vergleichbaren Zeitpunkten durchgeführt.

In beiden Therapiegruppen zeigte sich zu nahezu allen Zeitpunkten eine signifikant schlechtere Exekutivfunktion als
\end{abstract}

in der Vergleichsgruppe. Eine Verschlechterung von Arbeitsgedächtnis und Konzentration wurde in den ersten 6 Monaten der Anastrozol-Therapie sowohl unter der Kombination ( $\mathrm{p}<0,0001$ bzw. $\mathrm{p}<0,0009)$ als auch unter Anastrozol allein beobachtet ( $\mathrm{p}=0,0008 \mathrm{bzw}$. $\mathrm{p}=0,0002$ ). Dem folgte in beiden Gruppen eine Phase der Besserung. In der Gruppe mit alleinigem Anastrozol zeigte sich 12-18 Monate nach Therapiebeginn eine erneute Abnahme von Arbeitsgedächtnis und Konzentration ( $p<0,0001$ bzw. $p=0,02)$. Die längerfristigen Auswirkungen von Anastrozol auf die kognitive Leistungsfähigkeit über die 18-monatige Studiendauer hinaus müssen noch bestimmt werden.

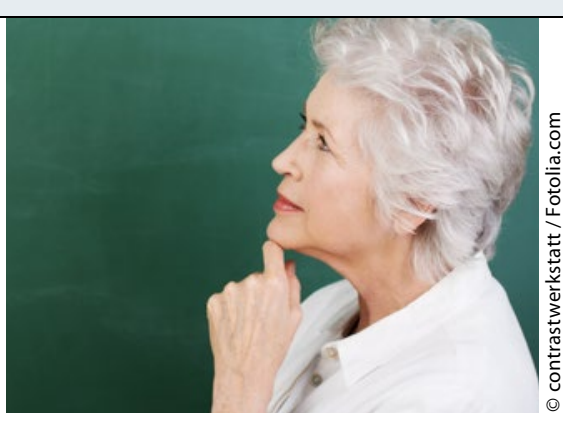

Durch eine Anastrozol-Therapie sind Arbeitsgedächtnis und Konzentration beeinträchtigt.

Fazit: Beginnend mit der Einnahme von Anastrozol (mit und ohne Chemotherapie) zeigte sich bei den postmenopausalen Frauen mit frühem Brustkrebs eine Abnahme von Arbeitsgedächtnis und Konzentration.

Frauen, die allein Anastrozol erhielten, erfuhren eine zweite Verschlechterung 12 bis 18 Monate nach Behandlungsbeginn. Die Einbußen in den Exekutivfunktionen scheinen bei den Brustkrebspatientinnen unabhängig zu sein von der systemischen Therapie.

Brigitte Schalhorn

Bender CM et al. Patterns of change in cognitive function with anastrozole therapy. Cancer. 2015; 121(15):2627-36.

\section{Mammakarzinom: Ausgewählte Subgruppe profitiert von adjuvanter HER2-Blockade}

\begin{abstract}
Etwa $20 \%$ der Frauen mit einem invasiven Mammakarzinom haben einen HER2-positiven Tumortyp. In einer Metaanalyse randomisierter Studien wurde der Nutzen einer adjuvanten, gegen HER2 gerichteten Antikörpertherapie bei kleinen Läsionen bestätigt.
\end{abstract}

U nter Patientinnen mit HER2-positivem Brustkrebs spricht insbesondere eine Untergruppe mit kleinen Läsionen $(\leq 2 \mathrm{~cm})$ günstig auf eine Trastuzumab-/Chemotherapie mit oder ohne Anti-Hormonbehandlung an. In einer Metaanalyse individueller Patientinnendaten wurde die Wirksamkeit einer adjuvanten Trastuzumab- mit der einer Standardtherapie ohne den monoklonalen anti-HER2-Antikörper bei solchen Patientinnen verglichen. Dazu berücksichtigten die Autoren 5 der 6 großen randomisierten Studien zur adjuvanten Therapie mit Trastuzumab. Wirksamkeits-Endpunkte waren krankheitsfreies
(DFS) und Gesamtüberleben (OS) . Stratifiziert wurde nach dem Hormonrezeptorstatus (HR-positiv/-negativ).

Insgesamt gingen die Daten von 4.220

Patientinnen mit HER2-positiven Tumoren von maximal $2 \mathrm{~cm}$ Durchmesser in die Analyse ein. 2.588 erhielten adjuvant Trastuzumab, 94\% der 2.263 Patientinnen mit HER2-positiven Tumoren hatten eine endokrine Therapie erhalten. Die kumulative Progress- oder RezidivInzidenz nach median 8 Jahren lag in der Gruppe der HR-positiven, mit Trastuzumab Behandelten bei 17,3\% gegenüber $24,3 \%$ bei Patientinnen ohne Trastuzumab, entsprechend einer Verbesse- rung im DFS um 7\%. Die Auswertung zum OS ergab einen Vorteil von 3,8\%. HR-positive Patientinnen mit NodalStatus N0 oder N1 profitierten stärker, Patientinnen mit HR-negativen Tumoren generell weniger von der adjuvanten Behandlung; in allen Fällen war die Trastuzumab-Gabe aber überlegen.

Fazit: Frauen mit einem HER2-positiven Mammakarzinom von geringer Größe profitierten „substanziell“ von einer adjuvanten Therapie mit Trastuzumab, so die Forscher. Patientinnen mit HR-positiver Erkrankung und Nodalstatus N0/ N1 könnten als Kandidatinnen für die Teilnahme an Studien infrage kommen, in denen weniger aggressive Therapieoptionen für diese frühen Tumorstadien untersucht werden. Wolfgang Zimmermann

O'Sullivan CC et al. Efficacy of adjuvant trastuzumab for patients with human epidermal growth factor receptor 2-positive early breast cancer and tumors $\leq 2 \mathrm{~cm}$ : a meta-analysis of the randomized trastuzumab trials. J Clin Oncol. 2015;33(24):2600-8. 